Commun. math. Phys. 35, 173-179 (1974)

(C) by Springer-Verlag 1974

\title{
Dilation-Analyticity and Decay Properties of Interactions
}

\author{
D. Babbitt ${ }^{\star}$ and E. Balslev ${ }^{\star} \star$ \\ Department of Mathematics, University of California, Los Angeles, California, USA
}

Received July 16, 1973

Abstract. Let $H=H_{0}+V$ be a Schrödinger operator on $L^{2}\left(\mathbb{R}^{n}\right)$. We show that the more dilation analytic $V$ is, the slower it must decay at infinity.

\section{Introduction}

In the theory of the Schrödinger operator $H=H_{0}+V$, various assumptions are made about the interaction $V$ in order to be able to prove useful theorems about the spectral and scattering properties of the operator. Two assumptions which are often made are dilation analyticity assumptions (see [1] and [2]) and decay assumptions (see, for example, [4]). These usually have not occurred together (at least explicitly). It is the purpose of this paper to explore the interrelations between these two assumptions. In particular we will show that the more dilation analytic $V$ is, the slower it must decay at infinity.

Our proof is based on a certain complex variable result (Lemma 3.2) which gives a sufficient condition for an analytic function defined in an angular sector to be 0 . This is a consequence of the Phragmen Lindelöf theorem and a theorem of Carlson.

\section{The Main Theorem}

We will denote by $\mathscr{H}$, the Hilbert space $L^{2}\left(R^{n}\right)$ of complex square integrable functions on $\mathbb{R}^{n}$. As usual, the inner product is defined by:

$$
\left(\psi_{1}, \psi_{2}\right)=\int_{\mathbb{R}^{n}} \overline{\psi_{1}(x)} \psi_{2}(x) d x .
$$

Also $\|\psi\|^{2} \equiv(\psi, \psi) . \mathscr{H}_{+}$will denote the completion of $C_{0}^{\infty}\left(\mathbb{R}^{n}\right)$ with respect to the norm $\|\psi\|_{+} \equiv\left\|H_{0} \psi\right\|+\|\psi\|$ where $H_{0}$ is the usual self-adjoint

$\star, \star \star$ This Research was partially supported by $\star$ NSF grant no. GP-33696 X and $\star \star$ NSF grant no. GP-36336. 
operator on $\mathscr{H}$ corresponding to the negative Laplacian

$$
-\Delta=-\sum_{j=1}^{n}\left(\frac{\partial}{\partial x^{j}}\right)^{2} \text {. }
$$

Note $\mathscr{H}_{+} \subseteq \mathscr{H}, \mathscr{B}\left(\mathscr{H}_{+}, \mathscr{H}\right)$ will denote the space of bounded linear mappings from $\mathscr{H}_{+}$to $\mathscr{H} \cdot \| \cdot|| \mid$ will denote the usual norm in $\mathscr{B}\left(\mathscr{H}_{+}, \mathscr{H}\right)$. Most interactions of physical interest in nonrelativistic quantum mechanics are elements of $\mathscr{B}\left(\mathscr{H}_{+}, \mathscr{H}\right)$ for $n=3$.

Let $\mathbb{R}^{+}$denote the multiplicative group of positive real numbers. Let $U(\varrho)$ be the unitary representation of $R^{+}$on $\mathscr{H}$ defined by

$$
(U(p) \varphi)(x) \equiv \varrho^{n / 2} \varphi(\varrho x)
$$

for $\varrho \in \mathbb{R}^{+}, \varphi \in \mathscr{H} .\left\{U(\varrho): \varrho \in \mathbb{R}^{+}\right\}$is the dilation group on $\mathscr{H}$. For later use, we will need the following lemma.

Lemma 2.1. Let $\psi \in C_{0}^{\infty}\left(\mathbb{R}^{n}\right)$ and $\varrho \in \mathbb{R}^{+}$. Then

$$
\left\|U\left(\varrho^{-1}\right) \psi\right\|_{+1}=\frac{1}{\varrho^{2}}\left\|H_{0} \psi\right\|+\|\psi\| .
$$

In particular,

$$
\left\|U\left(\varrho^{-1}\right) \psi\right\|_{+1} \leqq\|\psi\|_{+1} \quad \text { for } \quad \varrho \geqq 1 .
$$

Proof. A direct computation shows

This implies

$$
U(\varrho) H_{0} U\left(\varrho^{-1}\right) \psi=\varrho^{-2} H_{0} \psi .
$$

$$
\left\|H_{0} U\left(\varrho^{-1}\right) \varphi\right\|=\varrho^{-2}\left\|H_{0} \psi\right\|
$$

which, together with the unitarity of $U\left(\varrho^{-1}\right)$ on $\mathscr{H}$, implies (2.1).

Let $\mathscr{R}$ be the Riemann surface for $\ln z$. Thus $\mathscr{R}=\{(\varrho, \varphi) \mid \varrho>0, \varphi \in \mathbb{R}\}$ with the analytic structure defined by the function $\Psi(\varrho, \varphi)=\varrho e^{i \varphi}$. Let $0<\theta \leqq \infty$ and let $S_{\theta}=\{(\varrho, \varphi) \in \mathscr{R}:-\theta<\varphi<-\theta\} . S_{\theta}$ is called the $\theta$-sector in $\mathscr{R}$. When $\theta<\pi$, we identify $S_{\theta}$ with the obvious sector in $\mathbb{C}$.

Definition. Set $V \in \mathscr{B}\left(\mathscr{H}_{+1}, \mathscr{H}\right)$ and let $V(\varrho)=U(\varrho) V U\left(\varrho^{-1}\right)$. [It follows from Lemma 2.1 that $V(\varrho) \in \mathscr{B}\left(\mathscr{H}_{+}, \mathscr{H}\right)$.] We say $V$ is $S_{\theta}$ dilation analytic if $V(\varrho)$ can be continued to an $\mathscr{B}\left(\mathscr{H}_{+}, \mathscr{H}\right)$-valued analytic function in $S_{\theta}$. The potential $V$ is said to be exactly $S_{\theta}$-analytic if $V$ is $S_{\theta}$-analytic but not $S_{\theta+\varepsilon}$-analytic for any $\varepsilon>0$.

Examples. A function $V$ with compact support, viewed as a multiplication operator, is not dilation analytic at all. A Yukawa potential $V=\frac{e^{-k r}}{r}(n=3)$ is exactly $S_{\pi / 2}$ analytic. A Coulomb potential $V=\frac{1}{r}$ $(n=3)$ is $S_{\infty}$-analytic. 
Remark 2.2. A key property of an $S_{\theta}$ dilation analytic $V$ is the obvious

$$
V\left(\varrho e^{i \varphi}\right)=U(\varrho) V\left(e^{i \varphi}\right) U\left(\varrho^{-1}\right)
$$

for $-\theta<\varphi<\theta$. [We have abused notation by writing $V\left(\varrho e^{i \varphi}\right)$ rather than $V(\varrho, \varphi)$. No confusion should arise.]

We can now state our main theorem.

Theorem 2.3. Set $V \in \mathscr{B}\left(\mathscr{H}_{+}, \mathscr{H}\right)$ and let $\alpha>0$ be such that

1. $V$ is $S_{\theta}$-dilation analytic for some $\theta>-\frac{\pi}{2 \alpha}$.

2. $e^{k|x|^{\alpha}} V \in \mathscr{B}\left(\mathscr{H}_{+}, \mathscr{H}\right)$ for some $k>0$.

Then $V=0$.

The theorem says essentially that if $V$ is $S_{\theta}$ dilation analytic for some $\theta>\frac{\pi}{2 \alpha}$, then $V$ must "decay more slowly" than $e^{-k|x|^{\alpha}}$ for any $k>0$. The theorem is in some sense sharp since for $n \geqq 2, V=\frac{e^{-k|x|^{\alpha}}}{r} \in \mathscr{B}\left(\mathscr{H}_{+}, \mathscr{H}\right)$ $k>0, \alpha>0$ and $e^{k|x|^{\alpha}} V \in \mathscr{B}\left(\mathscr{H}_{+}, \mathscr{H}\right)$ and $V$ is $S_{\theta}$-dilation analytic for all $\theta<\frac{\pi}{2 \alpha}$.

It seems difficult, however, to formulate a necessary and sufficient condition for a potential to be exactly dilation - analytic in the angle $\frac{\pi}{2 \alpha}<\varphi<\frac{\pi}{2 \alpha}$.

For example, the function $e^{-r}\left(1+\sin r^{2}\right)$ decays as $e^{-r}$, but is dilation analytic in the angle $-\frac{\pi}{4}<\varphi<\frac{\pi}{4}$.

\section{An Application of the Phragmen - Lindelöf Theorem}

In this section we prove a result from complex function theory on which the proof of our main theorem is based.

Let us first state the following corollary of the Phragmen - Lindelöf theorem.

Lemma 3.1. Suppose that the function $f(z)$ is analytic in an angular sector

$$
\left\{z=\varrho e^{i \varphi} \mid \varphi_{1} \leqq \varphi \leqq \varphi_{2}, 1 \leqq \varrho<\infty\right\}
$$

where $\varphi_{2}-\varphi_{1}<\pi$, and that $f(z)$ satisfies the estimates for some $K, b, c>0$, 
(i) $\left|f\left(\varrho e^{i \varphi_{i}}\right)\right| \leqq K e^{-b \varrho} \quad$ for $\quad 1 \leqq \varrho<\infty, \quad i=1,2$,

(ii) $\left|f\left(\varrho e^{i \varphi}\right)\right| \leqq K e^{c \varrho}$, for $\varphi_{1}<\varphi<\varphi_{2}, 1 \leqq \varrho<\infty$.

\section{Then}

$\left(f\left(\varrho e^{i \varphi}\right) \mid \leqq K_{1} e^{-b \varrho}\right.$ for $\varphi_{1} \leqq \varphi \leqq \varphi_{2}, \quad 1<\varrho<\infty$, and some $K_{1}>0$.

Proof. We can assume that $\varphi_{1}=-\varphi_{0}, \varphi_{2}=\varphi_{0}, 0<\varphi_{0}<\frac{\pi}{2}$. Consider the function

$$
g(z)=e^{\frac{b}{\cos \varphi_{0}} z} f(z) .
$$

This function satisfies the estimates

(i) $\left|g\left(\varrho e^{i \varphi_{\imath}}\right)\right| \leqq K, \quad$ for $1 \leqq \varrho<\infty, \quad i=1,2$.

(ii) $\left|g\left(\varrho e^{i \varphi}\right)\right| \leqq K e^{\left(b \frac{\cos \varphi}{\cos \varphi_{0}}+c\right)_{\varrho}}$ for $-\varphi_{0}<\varphi<\varphi_{0}, \quad 1 \leqq \varrho<\infty$.

By the Phragmen - Lindelöf theorem ([6], p. 177),

$$
\left|g\left(\varrho e^{i \varphi}\right)\right| \leqq K_{1} \text { for }-\varphi_{0} \leqq \varphi \leqq \varphi_{0}, \quad 1 \leqq \varrho<\infty
$$

where $K_{1}=\max \left\{K, K_{2}\right\}, K_{2}=\max _{-\varphi_{0} \leqq \varphi \leqq \varphi_{0}}\left|g\left(e^{i \varphi}\right)\right|$.

This implies

$\left|f\left(\varrho e^{i \varphi}\right)\right| \leqq K_{1}^{-b \frac{\cos \varphi}{\cos \varphi_{0}} \varrho} \leqq K_{1} e^{-b \varrho} \quad$ for $\quad-\varphi_{0} \leqq \varphi \leqq \varphi_{0}, \quad 1 \leqq \varrho<\infty$.

By means of Lemma 3.1 we can now prove

Lemma 3.2. Suppose that the function $h\left(\varrho e^{i \varphi}\right)$ is analytic in the angular sector $\left\{\varrho e^{i \varphi} \mid-\varphi_{0} \leqq \varphi \leqq \varphi_{0}, 0<\varrho<\infty\right\}$ where $\frac{\pi}{2}<\varphi_{0}<\pi$, and that $h\left(\varrho e^{i \varphi}\right)$ satisfies the estimates

(i) $|h(\varrho)| \leqq K e^{-a_{1} \varrho}$ for $1<\varrho<\infty$,

(ii) $\left|h\left(\varrho e^{i \varphi}\right)\right| \leqq K e^{a_{2} \varrho} \quad$ for $\quad 0<|\varphi|<\varphi_{0}, \quad 1 \leqq \varrho<\infty$,

where $0 \leqq a_{2}<a_{1}\left|\cos \varphi_{0}\right|$.

Then $h\left(\varrho e^{i \varphi}\right) \equiv 0$.

Proof. Choose $\varepsilon$ and $\varphi_{1}, 0<\varepsilon<a_{1}, \frac{\pi}{2}<\varphi_{1}<\varphi_{0}$, such that

$$
-a=\left(a_{1}-\varepsilon\right) \cos \varphi_{1}+a_{2}<0 .
$$

Consider the function

$$
g(z)=e^{\left(a_{1}-\varepsilon\right) z} h(z) .
$$

The function $g(z)$ satisfies the estimates

(i') $\mid g(\varrho) \leqq K e^{-\varepsilon \varrho} \quad$ for $\quad 1 \leqq \varrho<\infty$, 
(ii') $\left|g\left(\varrho e^{i \varphi}\right)\right| \leqq K e^{\left[\left(a_{1}-\varepsilon\right) \cos \varphi+a_{2}\right] \varrho}$ for $\quad 0<|\varphi| \leqq \varphi_{1}, \quad 1 \leqq \varrho<\infty$, in particular

(iii') $\left|g\left(\varrho e^{-+i \varphi_{0}}\right)\right| \leqq K e^{-a \varrho}$.

Applying Lemma 3.1 to the function $g\left(\varrho e^{i \varphi}\right)$ on the sector

$$
\left\{\varrho e^{i \varphi} \mid 0 \leqq \varphi \leqq \varphi_{1}, 1 \leqq \varrho<\infty\right\} \quad \text { and } \quad\left\{\varrho e^{i \varphi} \mid-\varphi_{1} \leqq \varphi \leqq 0,1 \leqq \varrho<\infty\right\}
$$

we obtain

$$
\left|g\left(\varrho e^{i \varphi}\right)\right| \leqq K e^{-b \varrho} \text { for } \quad-\varphi_{1} \leqq \varphi \leqq \varphi_{1}, \quad 1 \leqq \varrho<\infty,
$$

where $b=\min \{\varepsilon, a\}$.

An application of Carlson's theorem ([5], p. 185) or [3], Lemma 1.2 ${ }^{1}$, now implies $g\left(\varrho e^{i \varphi}\right) \equiv 0$ and hence $h\left(\varrho e^{i \varphi}\right) \equiv 0$.

\section{Proof of the Main Theorem}

Proof of the Theorem for $\alpha=1$. Let $\hat{\mathscr{D}}$ be the set of functions $\psi \in C_{0}^{\infty}\left(\mathbb{R}^{n}\right)$ such that $\operatorname{supp} \psi \subset\left\{x \in R^{n}|| x \mid \geqq \varepsilon\right\}$ for some $\varepsilon=\varepsilon_{\psi}>0$. $\hat{\mathscr{D}}$ is dense in $\mathscr{H}$. Thus it is sufficient to show that the analytic function

$$
F_{\psi, \xi}(z) \equiv(\psi, V(z) \xi) \quad z=\varrho e^{i \varphi}, \quad z \in S_{\theta},
$$

is identically equal to 0 for all $\psi \in \hat{\mathscr{D}}, \xi \in C_{0}^{\infty}\left(\mathbb{R}^{n}\right)$. We will show that $F_{\psi, \xi}\left(\varrho e^{i \varphi}\right)$ satisfies the hypothesis of Lemma 3.2 for $K_{3}=0$.

Let $\varphi_{0}$ be such that $\pi / 2<\varphi_{0}<\theta, \varphi_{0}<\pi$, and let

$$
\operatorname{supp}(\psi) \subseteq\left\{x \in R^{n}|| x \mid \geqq \varepsilon\right\}, \quad \varepsilon>0 .
$$

Then, for $\varrho \geqq 1,-\varphi_{0} \leqq \varphi \leqq \varphi_{0}$, we have:

$$
\begin{aligned}
\left|F_{\psi, \xi}\left(\varrho e^{i \varphi}\right)\right| & =\left|\left(\psi, U(\varrho) V\left(e^{i \varphi}\right) U\left(\varrho^{-1}\right) \xi\right)\right| \\
& =\left|\left(U\left(\varrho^{-1}\right) \psi, V\left(e^{i \varphi}\right) U\left(\varrho^{-1}\right) \xi\right)\right| \\
& \leqq\|\psi\|_{2}\|\xi\|_{+} \sup _{-\varphi_{0} \leqq \varphi \leqq \varphi_{0}} \mid\left\|V\left(e^{i \varphi}\right)\right\|=K<\infty .
\end{aligned}
$$

We have used (2.2), (2.3), the unitarity of $U\left(\varrho^{-1}\right)$ on $\mathscr{H}$, and the fact that $\varphi \leadsto V\left(e^{i \varphi}\right)$ is continuous from $\left[-\varphi_{0}, \varphi_{0}\right]$ to $\mathscr{B}\left(\mathscr{H}_{+}, \mathscr{H}\right)$. This shows that (3.2) is satisfied for $K_{3}=0$ and $K=K_{1}$.

To see the exponential decay along the positive real axis we need the following lemma.

1 Titchmarsh's statement of Carlson's theorem assumes that $f(Z)$ is regular for $\operatorname{Re} z \geqq 0$. However, his proof only requires that $f$ be regular for $\operatorname{Re} z \geqq 0,|z| \geqq r_{0}$ for some $r_{0}>0$ (see [5], p. 182). Similarly the proof of [3], Lemma 1.2, is obviously valid if $\left\{z|| z \mid<r_{0}\right\}$ is delated from the domain of the function. 
Lemma 4.1. Let $\psi \in \hat{\mathscr{D}}$ be such that $\operatorname{supp} \psi \subset\{x|| x \mid \geqq \varepsilon\}$. Let $k, \alpha>0$. Then

$$
\left\|e^{-k|x|^{\alpha}} U\left(\varrho^{-1}\right) \psi\right\| \leqq e^{-k \varepsilon^{\alpha} \varrho^{\alpha}}\|\psi\|, \quad \varrho \geqq 1 .
$$

Proof. First of all, note that $\operatorname{supp}\left(U\left(\varrho^{-1}\right) \psi\right) \subseteq\left\{x \in R^{n}|| x \mid \geqq \varrho \varepsilon\right\}$. Then

$$
\begin{aligned}
& \left\|e^{-k|x|^{\alpha}} U\left(\varrho^{-1}\right) \psi\right\|^{2} \\
& \quad=\int_{\mathbb{R}^{n}} e^{-2 k|x|^{\alpha}}\left|U\left(\varrho^{-1}\right) \psi\right|^{2} d x \\
& \quad=\int_{|x| \geqq \varrho \varepsilon} e^{-2 k|x|^{\alpha}}\left|U\left(\varrho^{-1}\right) \psi\right|^{2} d x \\
& \quad \leqq e^{-2 k \varrho \varepsilon^{\alpha} \varrho^{\alpha}} \int\left|U\left(\varrho^{-1}\right) \psi\right|^{2} d x \\
& \quad \leqq e^{-2 k \varepsilon^{\alpha} \varrho^{\alpha}}\left\|U\left(\varrho^{-1}\right) \psi\right\|^{2} \\
& \quad=e^{-2 k \varepsilon^{\alpha} \varrho^{\alpha}}\|\psi\|^{2}
\end{aligned}
$$

which establishes (4.2).

Applying this lemma to $F_{\psi, \xi}(z)$ we have:

$$
\begin{aligned}
\left|F_{\psi, \xi}(\varrho)\right| & =\left|\left(\psi, U(\varrho) V U\left(\varrho^{-1}\right) \xi\right)\right| \\
& =\left|\left(e^{-k|x|} U\left(\varrho^{-1}\right) \psi, e^{i|x|} V U\left(\varrho^{-1}\right) \xi\right)\right| \\
& \leqq\left(\left\|\mid e^{k|x|} V\right\|\|\| \psi\|\| \xi \|_{+}\right) e^{-k \varepsilon \varrho} .
\end{aligned}
$$

Thus letting $k \varepsilon=K_{2}, H_{3}=0, K_{1}=\max \left(K,\left\|e^{k|x|} V\right\|\|\psi\|\|\xi\|_{+}\right)$, and using (4.1) and (4.3) we see the hypotheses of Theorem 3.1 are satisfied and therefore $F_{\psi, \xi} \equiv 0$ on $S_{\theta}$.

Proof of the Theorem for $\alpha>0$.

As before, it is sufficient to show that $F_{\psi, \xi}(z) \equiv(\psi, V(z) \xi)$ is identically 0 for $\psi \in \hat{\mathscr{D}}, \xi \in C_{0}^{\infty}\left(\mathbb{R}^{n}\right), z \in S_{\theta}$. First of all we can show in exactly the same way as above, that there exists $K_{1}, K_{2}>0$ such that

and

$$
\left|F_{\psi, \xi}\left(\varrho e^{i \varphi}\right)\right| \leqq K_{1}, \quad \varrho \geqq 1, \quad-\theta<\varphi<\theta
$$

$$
\left|F_{\psi, \xi}(\varrho)\right| \leqq K_{1} e^{-K_{2} \varrho^{\alpha}}, \quad \varrho \geqq 1 .
$$

Consider the mapping $f: S_{\alpha \theta} \rightarrow S_{\theta}:(\varrho, \varphi) \leadsto\left(\varrho^{\alpha^{-1}}, \alpha^{-1} \varphi\right)$. This is clearly an analytic bijection. Let $G=F_{\psi, \xi} \circ f$. This is an analytic function on $S_{\alpha \theta}$ and note $\alpha \theta>\pi / 2$. Moreover (4.4) and (4.5) imply

and

$$
\left|G\left(\varrho e^{i \varphi}\right)\right| \leqq K_{1}, \quad \varrho \geqq 1, \quad-\alpha \theta<\varphi<\alpha \theta
$$

$$
|G(\varrho)| \leqq K_{1} e^{-K_{2} \varrho}, \quad \varrho \geqq 1 .
$$

Applying Theorem 3.1 with $\pi / 2<\varphi_{0}<\alpha \theta, \varphi_{0}<\pi K_{1}, K_{2}$ as in (4.6) and (4.7) and, $K_{3}=0$, we see $G \equiv 0$ or $S_{\alpha \theta}$. This implies $G \circ f^{-1}=F_{\psi, \xi} \equiv 0$ on $S_{\theta}$. 


\title{
References
}

1. Aguilar, J., Combes, J. M.: Commun. math. Phys. 22, 269-279 (1971)

2. Balslev, E., Combes, J. M.: Commun. math. Phys. 2, 280-294 (1971)

3. Balslev, E.: Absence of positive eigenvalues of Schrödinger operators. Preprint UCLA 1973, submitted for publication

4. Shenk,E., Thoe,D.: Rocky Mountain J. Math. 1, 1 (1971)

5. Titchmarsh,E.C.: The Theory of Functions. Oxford: University Press 1939

\author{
D. Babbitt \\ E. Balslev \\ Department of Mathematics \\ University of California \\ Los Angeles, Cal. 90024, USA
}


